\title{
A Carbon-rich Mira variable in a globular cluster: A stellar merger
}

\section{J. Menzies*}

South African Astronomical Observatory, P.O. Box 9 Observatory, Cape Town, South Africa

E-mail: wmesaao.ac.za

\section{M.W. Feast}

South African Astronomical Observatory, P.O. Box 9 Observatory, Cape Town, South Africa

\begin{abstract}
The membership of Matsunaga's variable 1, a carbon-rich, mass-losing, Mira variable, in the globular cluster Lynga 7 is discussed on the basis of radial velocities. We conclude that it is a member, the first known C-Mira in a globular cluster. Since such a variable is expected to have an age of $\sim 1-2$ Gyr and an initial mass of $\sim ! 1.5$ solar masses, we conclude that this star must be the product of a stellar merger.
\end{abstract}

SALT Science Conference 2015,

1-5 June 2015

Stellenbosch Institute of Advanced Study, South Africa

*Speaker. 
The full details of this work are published in [1]

\section{References}

[1] Feast, M. W., Menzies, J. W., \& Whitelock, P. A. 2013, MNRAS, 428, L36 